\title{
Electron Charged States of Pt-silicide Nanodots as Evaluated by Using an AFM/Kelvin Probe Technique
}

\author{
Katsunori Makihara, Kazuhiro Shimanoe, Mitsuhisa Ikeda, Akio Ohta, Seiichiro Higashi \\ and Seiichi Miyazaki \\ Graduate School of Advanced Sciences of Matter, Hiroshima University \\ Kagamiyama 1-3-1, Higashi-Hiroshima 739-8530, Japan \\ Fax: 81-82-424-7548, e-mail: semicon@hiroshima-u.ac.jp
}

\begin{abstract}
We formed high density platinum-silicide nanodots on an ultrathin $\mathrm{SiO}_{2}$ layer and characterized their electronic charged states by an AFM/Kelvin probe technique. A $\sim 1.8-\mathrm{nm}$-thick Pt film deposited on pre-grown $\mathrm{Si}$ quantum dots (Si-QDs) with an areal dot density of $\sim 1.2 \times 10^{11} \mathrm{~cm}^{-2}$ was exposed to remote hydrogen plasma. The formation of platinum-silicide nanodots with an areal dot density of $\sim 1.2 \times 10^{11} \mathrm{~cm}^{-2}$ was confirmed after examining the surface morphology and measuring the valence band spectra by AFM and XPS, respectively. The surface potential of the nanodots changed in a stepwise manner with respect to the tip bias due to multistep electron injection into and extraction from the platinum-silicide nanodots.
\end{abstract}

Key words: Platinum-silicide, Nanodots, Remote $\mathrm{H}_{2}$ Plasma, AFM/KFM

\section{INTRODUCTION}

The application of metal-based nanodots to a floating gate in MOS memories has been attracting much attention from a viewpoint of improvement in charge retention characteristics [1]. So far, we have reported the formation of $\mathrm{Ni}$ and $\mathrm{Pt}$ nanodots on $\mathrm{SiO}_{2}$ with an areal density as high as $10^{11} \mathrm{~cm}^{-2}$ by exposing ultrathin $\mathrm{Ni}$ and $\mathrm{Pt}$ films on $\mathrm{SiO}_{2}$ to remote $\mathrm{H}_{2}$ plasma at room temperature $[2,3]$. One of the major issues for the floating gate application of metal-based nanodots is to minimize metal diffusion into a gate dielectric layer [4] that is responsible for the degradation of oxide reliability and a large variation in the memory window. To achieve good thermal stability and long retention characteristics for electron and holes, Pt-silicide is a promising candidate. In this work, we extended our research work to form high density nanodots of PtSi on $\mathrm{SiO}_{2}$ and characterized their electronic charged states by using an AFM/Kelvin Force Microscopy (KFM).

\section{Experimental}

A $\sim 3.5 \mathrm{~nm}$-thick $\mathrm{SiO}_{2}$ was thermally-grown on p-Si(100). To obtain an $\mathrm{OH}$-terminated $\mathrm{SiO}_{2}$ surface which enables us to enhance Si-QD dot density and improve the dot size uniformity, the $\mathrm{SiO}_{2}$ layer was slightly etched by being dipped into the $0.1 \% \mathrm{HF}$ solution for $1 \mathrm{~min}$. Hemispherical single crystalline Si-QDs with $\sim 1.2 \times 10^{11} \mathrm{~cm}^{-2}$ in areal number density were self-assembled on OH-terminated $\mathrm{SiO}_{2}$ surface from the thermal decomposition of pure $\mathrm{SiH}_{4}$ [5]. Subsequently, Pt film as thin as $\sim 1.8 \mathrm{~nm}$ was deposited at room temperature by $\mathrm{Ar}$ suputtering and followed by exposure to remote VHF plasma of pure $\mathrm{H}_{2}$ at room temperature. The $\mathrm{H}_{2}$ plasma was generated inductively by supplying $60 \mathrm{MHz}$ power through an external single-turn antenna surrounding a quartz tube. The areal dot density was evaluated by atomic force microscopy (AFM). The chemical bonding features of the $\mathrm{Pt}$ film/Si-QDs were examined by X-ray photoelectron spectroscopy (XPS) measurements using a monochromatised AlKa source $(1486.71 \mathrm{eV})$. Also, electron injection to and extraction from PtSi nanodots were carried out by scanning the sample surface with an electrically-biased AFM probe tip in a tapping mode at room temperature in clean room air, where a Rh-coated $\mathrm{Si}_{3} \mathrm{~N}_{4}$ cantilever was used. Before and after electron injection or extraction, the topographic and corresponding surface potential images were simultaneously taken with a non-contact Kelvin-probe mode [6].

\section{Results and Discussions}

Figure 1 shows the AFM images taken at each process step. Before and after Pt deposition, no significant change in the dot density was observed. This result indicates conformal coverage of Si-QDs surface with the ultrathin Pt film. AFM images taken before and after remote $\mathrm{H}_{2}$ plasma treatment confirm almost no change in the dot density but a slight increase in dot size. The influence of remote $\mathrm{H}_{2}$ plasma treatment on the chemical bonding features of the $\sim 1.8$-nm-thick $\mathrm{Pt}$ film/Si-QDs after remote $\mathrm{H}_{2}$ plasma treatment (as shown in Fig. 1(d)) was evaluated by XPS. Figure 2(a) compares the valence band spectra taken after the remote $\mathrm{H}_{2}$ plasma exposure with the valence band spectra for $\mathrm{Pt}$ and $\mathrm{Pt}$-silicide (PtSi) film as references. We deconvoluted $\mathrm{SiO}_{2}$ and $\mathrm{Si}(100)$ spectra from measured spectra of $\mathrm{Pt} / \mathrm{Si}$-QDs/ $/ \mathrm{SiO}_{2} / \mathrm{Si}(100)$ as shown in Fig. 2(b). At near the top of the valence band spectra, after subtracting $\mathrm{SiO}_{2}$ and $\mathrm{Si}(100)$ components (Fig. 2 
(b)), the spectra shape for Pt/Si-QDs treated by remote $\mathrm{H}_{2}$ plasma is similar to those of a reference PtSi film with a composition of $\mathrm{Pt} /(\mathrm{Pt}+\mathrm{Si})=\sim 70 \%$. These results confirm the formation of Pt-silicide nanodots with a high areal density of $\sim 1.2 \times 10^{11} \mathrm{~cm}^{-2}$. In addition, from the cut-off energy for photoemission in lower kinetic energy scale, the work function value of the Pt-silicide nanodots was also evaluated to be $\sim 5.1 \mathrm{eV}$, being close to Pt-monosilicide as also expected from the analysis of core line spectra.

To confirm electrical isolation among the $\mathrm{PtSi}$ nanodots, before and after scanning an electrically-biased Rh-coated AFM tip on the sample surface, the topographic and corresponding surface potential images were simultaneously taken in a KFM as shown in Fig. 3. Without any bias applied to the sample surfaces, uniform surface potential image was observed (Fig. 3(a)). When the AFM tip was biased at $-2.0 \mathrm{~V}$ with respect to the substrate and scanned on the sample surface in this area, a decrease in the surface potential of corresponding area was observed (Fig. 3(b)). But, in the unbiased area, no change in the surface potential was detectable. Prior to the $\mathrm{H}_{2}$ plasma treatment for the Pt film on Si-QDs, no change in the surface potential was observable with applying tip biases because of an electrically well-conductive surface. These results indicate electron injection to the dot from $\mathrm{Rh}$ tip. By scanning with the tip biased at $+2.0 \mathrm{~V}$, the

\section{$3.0 \mathrm{~nm}$}
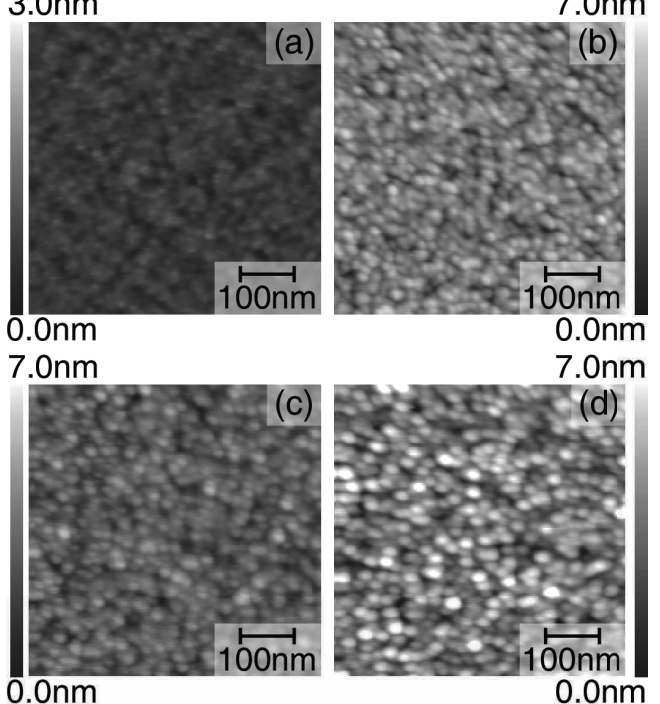

$0.0 \mathrm{~nm}$

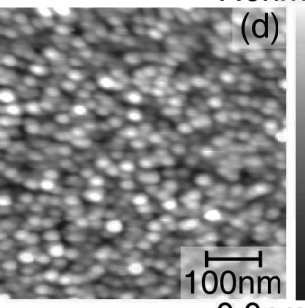

$0.0 \mathrm{~nm}$

Fig. 1 AFM images of $\sim 3.5$-nm thick as-grown $\mathrm{SiO}_{2}$ (a), $\mathrm{Si}-\mathrm{QDs} / \mathrm{SiO}_{2}$ (b), ultrathin $\mathrm{Pt}$ film deposited on Si-QDs before (c) and after remote $\mathrm{H}_{2}$ plasma treatment at VHF power of $350 \mathrm{~W}$ (d). Si-QDs with areal dot density of $\sim 1.2 \times 10^{11} \mathrm{~cm}^{-2}$ were formed on the $\mathrm{OH}$-terminated $\mathrm{SiO}_{2}$. $\mathrm{H}_{2}$ pressure, VHF power and substrate temperature were maintained at 0.26 Torr, 350 $\mathrm{W}$ and RT, respectively.

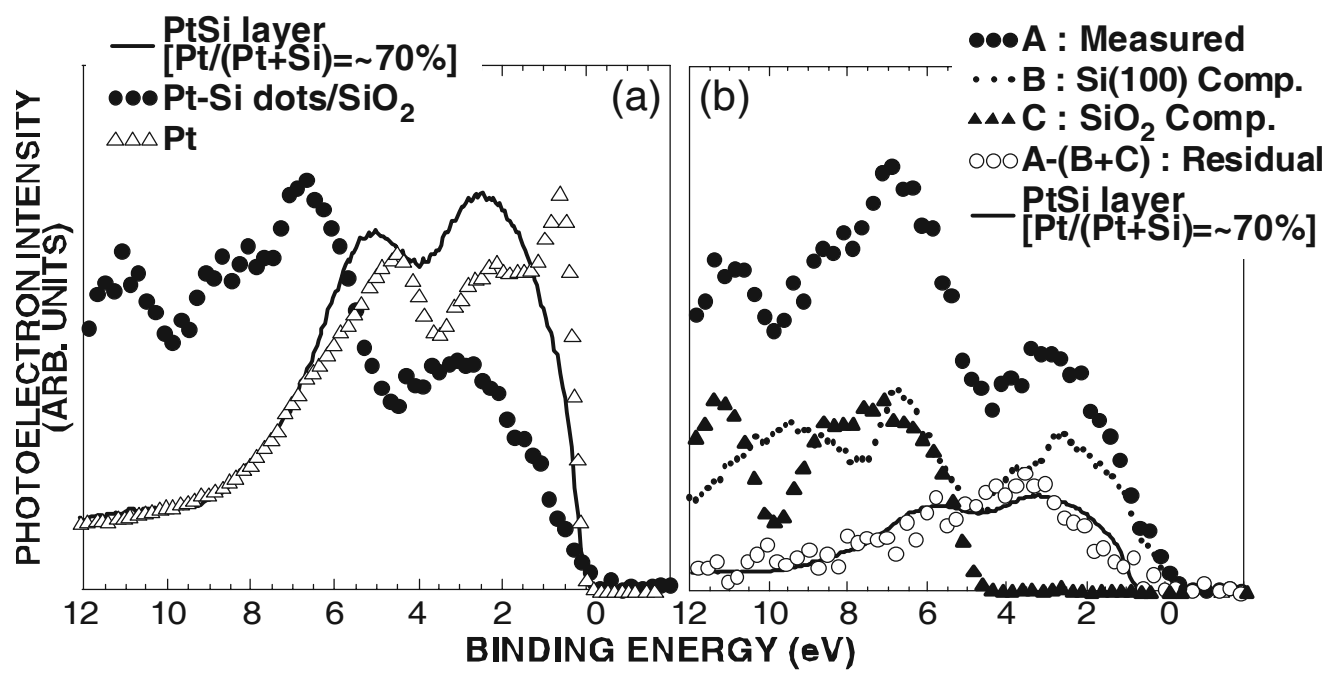

Fig. 2 Valence band spectra of $\mathrm{Pt} / \mathrm{Si}-\mathrm{QDs} / \mathrm{SiO}_{2}(\sim 3.5 \mathrm{~nm}) / \mathrm{Si}(100)$ treated with the remote $\mathrm{H}_{2}$ plasma. Valence band spectra of pure $\mathrm{Pt}$ and $\mathrm{PtSi}$ films with a composition of $\mathrm{Pt} /(\mathrm{Pt}+\mathrm{Si})=\sim 70 \%$ are also shown as references.
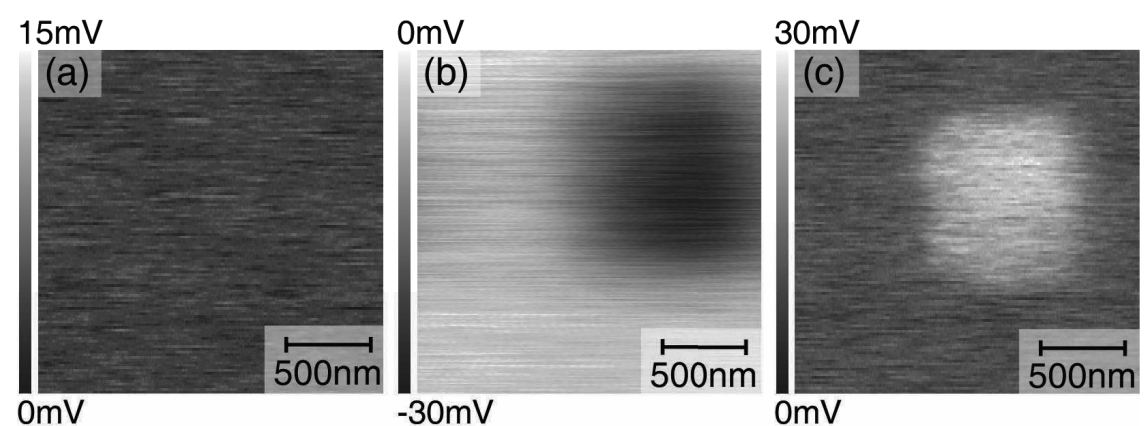

Fig. 3 Surface potential images measured in a Kelvin probe mode before (a) and after electron extraction and injection at tip biases of $-1.0 \mathrm{~V}(\mathrm{~b})$ and $+0.5 \mathrm{~V}(\mathrm{c})$, respectively. 


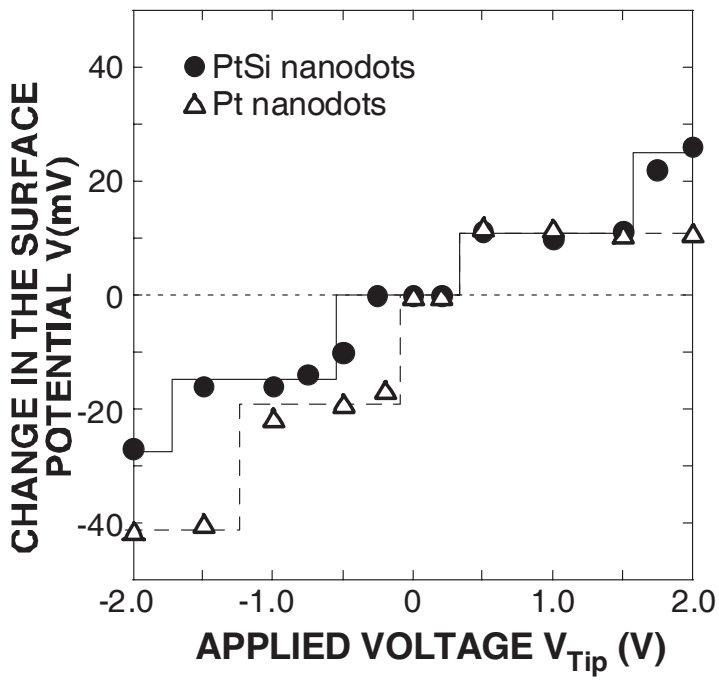

Fig. 4 Surface potential changes caused by electron extraction from and injection to the PtSi nanodots as a function of the applied tip bias.

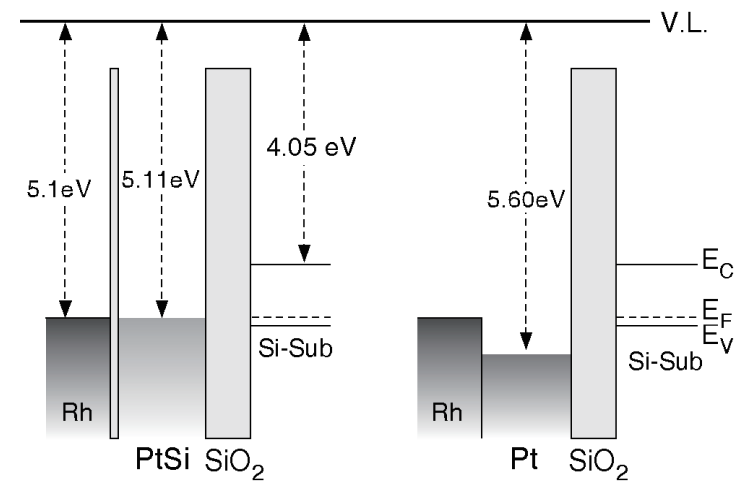

Fig. 5 Energy band diagrams of $\mathrm{PtSi}$ and $\mathrm{Pt}$ nanodots $/ \mathrm{SiO}_{2} / \mathrm{Si}(100)$. For the case of $\mathrm{PtSi}$ nanodots, the surface was covered with $\mathrm{Si}$ oxide due to native oxidation, which was confirmed from XPS spectra of Si2p.

surface potential raises (Fig. 3(c)). These surface potential changes with the plasma treatment indicate the migration of $\mathrm{Pt}$ atoms in between the dots to reveal the $\mathrm{SiO}_{2}$ surface. Changes in the surface potential as a function of applied tip biases were summarized as shown in Fig. 4. To compare the minimum tip bias for electron injection to the PtSi and $\mathrm{Pt}$ dots, we also measured change in the surface potential of Pt nanodot. At tip biases in the range from -2 to $+2 \mathrm{~V}$, the surface potential on the dots were changed in a stepwise manner due to electron injection to and emission from the nanodots. This result can be interpreted in terms of multistep electron charging and discharging characteristics of nanodots associated with their charging energy. The minimum tip biases for electron charging to $\mathrm{Pt}$ and PtSi nanodots were -0.2 and $-0.5 \mathrm{~V}$, respectively. The difference of minimum tip biases for electron injection to dots is attributed to the difference in the work function as schematically illustrated in Fig. 5.

We also measured charge retention characteristics of $\mathrm{PtSi}$ nanodots at room temperature in clean room air in comparison with Si-QDs with almost the same size as

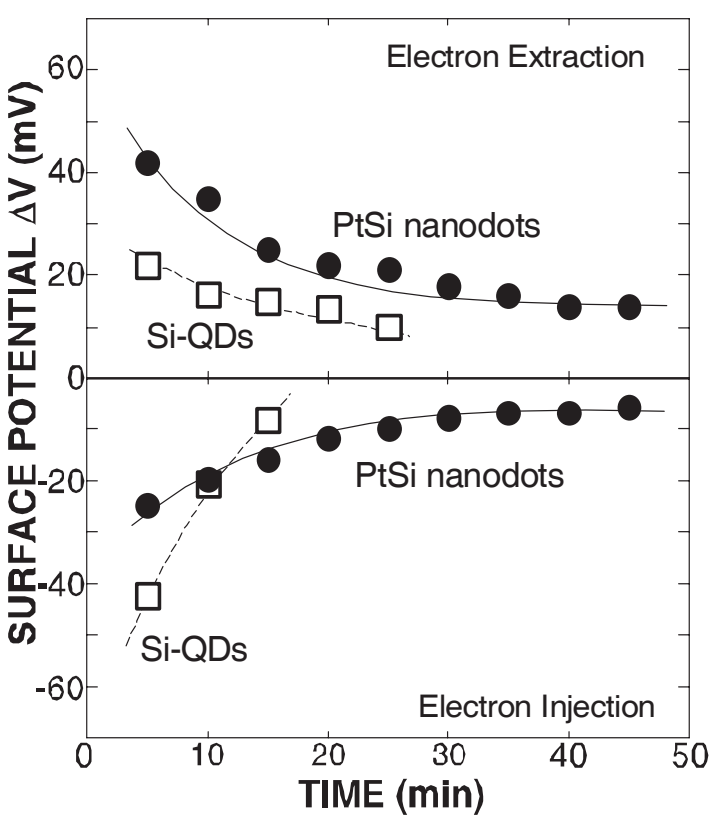

Fig. 6 Charge retention characteristics of $\mathrm{PtSi}$ nanodots and Si-QDs evaluated from changes in the surface potential after electron extraction from and injection to the dots at tip biases of +3.0 and $-3.0 \mathrm{~V}$, respectively.

PtSi nanodots as shown in Fig. 6. The retention characteristics were evaluated from the change in the surface potential generated by scanning the tip biased at +3.0 and $-3.0 \mathrm{~V}$. In the case of Si-QDs, the surface potentials for both charged states monotonously decay with time to almost neutral states. In contrast, for the case of PtSi nanodots, both positively and negatively charged states were stably retained in comparison to the case of Si-QDs. The observed difference in the retention characteristics between the $\mathrm{Si}-\mathrm{QDs}$ and $\mathrm{PtSi}$ dots can be interpreted in term of the difference in potential depth. The shorter retention time of the Si-QDs can be attributed to the holes recombination with electrons injected through the $\mathrm{SiO}_{2}$ and the electron tunneling from the $\mathrm{Si}$-QDs to the $\mathrm{Si}(100)$ substrate. In contrast, a deep potential well for both electrons and holes, which is originating from the work function of PtSi nanodots, is responsible for the stable retention of electrons and holes.

We also applied the PtSi nanodots to a floating gate (FG) MOS memory. For the fabrication of nanodots FG-MOS capacitors, after the formation of PtSi nanodots, a $\sim 27 \mathrm{~nm}$-thick oxide layer was grown as a control oxide by inductively-coupled remote plasma CVD with $\mathrm{SiH}_{4}$ and excited $\mathrm{O}_{2} / \mathrm{Ar}$ at a temperature of $350^{\circ} \mathrm{C}$. Figure 7 shows capacitance-voltage $(\mathrm{C}-\mathrm{V})$ characteristics of the MOS capacitor with the PtSi-nanodots FG measured with different ranges in gate voltage swing. The calculated ideal $\mathrm{C}-\mathrm{V}$ curve is also shown as a reference. After application of positive and negative gate biases, $\mathrm{C}-\mathrm{V}$ curve shows positive and negative flat-band voltage shifts from the calculated ideal $\mathrm{C}-\mathrm{V}$ curve, respectively. The positive flat-band voltage shift is due to electron injection to the nanodot FG through the tunnel oxide and the negative flat-band voltage shift is attributed to electron emission from the 


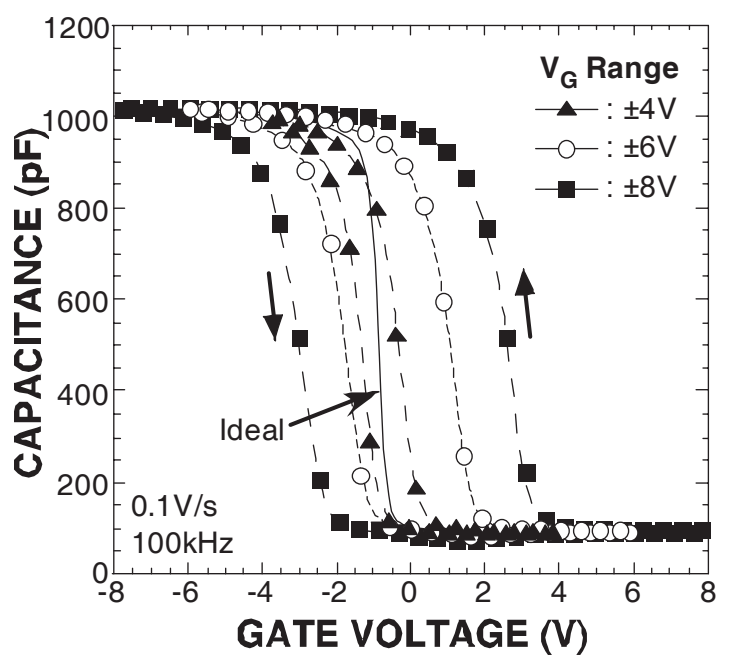

Fig. 7 Capacitance-voltage characteristics of a MOS capacitor with a PtSi nanodots floating gate measured at $100 \mathrm{kHz}$ with gate voltage sweep rate of $0.1 \mathrm{~V} / \mathrm{s}$.

nanodots FG. The flat-band voltage shifts as functions of maximum applied gate biases in each polarity were summarized as shown in Fig. 8. The positive flat-band shift monotonously increases with maximum gate bias. In contrast, the negative flat-band shift is suppressed at the maximum gate biases below $5 \mathrm{~V}$, reflecting the deep potential well of the Pt nanodots.

\section{Conclusions}

We fabricated platinum-silicide nanodots with an areal dot density of $\sim 1.2 \times 10^{11} \mathrm{~cm}^{-2}$ on a $\sim 3.5$-nm-thick $\mathrm{SiO}_{2} / \mathrm{p}-\mathrm{Si}(100)$ layer by the silicidation of self-assembled Si-QDs; the silicidation reaction was induced by remote $\mathrm{H}_{2}$ plasma treatment. The valence band spectra of $\mathrm{Pt} / \mathrm{Si}-\mathrm{QDs}$ obtained after exposure to remote $\mathrm{H}_{2}$ plasma indicated the formation of Pt-monosilicide dots. The change in the surface potential of the Pt-Si nanodots, which was measured in clean room air at room temperature by using an AFM/Kelvin probe technique, indicated multistep electron transfer, i.e. electron injection into and extraction from $\mathrm{Ni}-\mathrm{Si}$ nanodots, with respect to the tip bias. The remote $\mathrm{H}_{2}$ plasma treatment of ultrathin metal films deposited on Si-QDs is a very promising technique for the low temperature fabrication of metallic nanodots that can serve as charge storage nodes on $\mathrm{SiO}_{2}$ layers. These results provide useful information for the fabrication of high-performance FG memories.

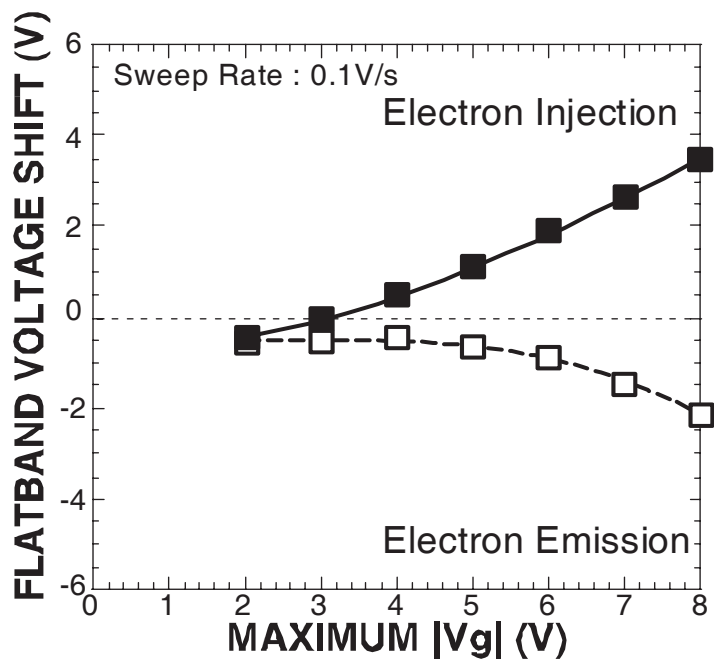

Fig. 8 The flat-band voltage shifts of MOS capacitors with a floating gate made of PtSi nanodots as functions of the maximum applied gate bias in each polarity in $\mathrm{C}-\mathrm{V}$ curves of Fig. 6 .

\section{Acknowledgements}

This research was supported in part by Grants-in Aid for Scientific Research (A) No. 18206035 and for Scientific Research on Priority Area (458) No. 18063017 from the Ministry of Education, Culture, Sports, Science and Technology, Japan.

6. References

[1] Z. Liu, C. Lee, V. Narayanan, G. Pei and E. C. Kan, IEEE Trans. Electron Devices 49 (2002) 1606.

[2] K. Makihara, K. Shimanoe, Y. Kawaguchi, M. Ikeda, S. Higashi and S. Miyazaki, Jpn. J. Appl. Phys. 47 (2008) 3099.

[3] K. Shimanoe, K. Shimanoe, K. Makihara, M. Ikeda, R. Matsumoto, S. Higashi and S. Miyazaki, Proc. of 2008 Asia-Pacific Workshop on Fundamentals and Applications of Advanced Semiconductor Devices, Hokkaido, 2008, pp. 77-80.

[4] J. T. Mayer, R. F. Lin and E. Garfunkel, Surf. Sci. 265 (1992) 102.

[5] S. Miyazaki, Y. Hamamoto, E. Yoshida, M. Ikeda and M. Hirose; Thin Solid Films 369 (2000) 55.

[6] K. Takeuchi, H. Murakami and S. Miyazaki; Proc. ECS Int. Semiconductor Technology Conf., Tokyo, 2002, p. 1.

(Received December 9, 2008; Accepted February 24, 2009) 